\title{
Efficient Computation of RNA Partition Functions Using McCaskill's Algorithm
}

\author{
Chunchun Zhao \\ Amadeus IT Group \\ Email: zhaochunchun@gmail.com
}

\author{
Sartaj Sahni \\ Department of Computer and Information \\ Science and Engineering \\ University of Florida \\ Email:sahni@cise.ufl.edu
}

\begin{abstract}
We develop efficient single- and multi-core algorithms to compute partition functions for RNA sequences. Our algorithms, which are based on McCaskill's algorithm, are benchmarked against state-of-the-art fast algorithms obtained using the parallelizing source-to-source compilers PLUTO and TRACO. On our Intel I9 computational platform, our best single core algorithm takes up to $81.2 \%$ less time than the single core algorithm resulting from PLUTO, which is faster than that obtained from TRACO. Our best multi-core algorithm takes up to $84.7 \%$ less time than the multi-core algorithm obtained using TRACO when run with 20 threads (our I9 has 10 cores and supports hyperthreading); the TRACO multi-core algorithm is faster than the PLUTO one.
\end{abstract}

\section{INTRODUCTION}

$\mathbf{S}$ EVERAL algorithms have been proposed to determine the minimum energy secondary structure of an RNA molecule. Smith and Waterman [1] and Nussinov et al. [2] have proposed a dynamic programming algorithm to maximize the number of complementary base pairs. These algorithms are oversimplified and do not generate good RNA secondary structure predictions. Zuker et al. [3] first defined five different loops that are basic units of the RNA secondary structure and proposed an energy model for each loop. Using this energy model, they developed a dynamic programming algorithm to determine the total minimum free energy structure for a given RNA sequence. The original approach of Zuker et al. [3] has been enhanced in [4], [5] by Zuker to handle more complex cases. However, Zuker's approach only provides a globally optimal secondary structure at equilibrium where each loop has been evaluated by the energy model without errors. To calculate the variance of the secondary structure, Zuker [6] proposed a further extension to calculate all suboptimal secondary structures of an RNA sequence. McCaskill [7] has proposed a totally different method to compute the full equilibrium partition function, which is the sum of the contributions of the suboptimal structures. McCaskill's algorithm computes the probabilities of each individual base pair of $(i, j)$ in the RNA sequence. These probabilities are used, for example by the software LocaRNA [8] and PMComp/PMMulti [9] for simultaneous folding and alignment and in algorithms to predict RNA structure with a maximum expected accuracy [10], [11].

Many parallel algorithms for RNA secondary structures have also been proposed. ( see, for e.g., [12], [13], [14],
[15], [16], [17], [18], [19], [20], [21], [22], [23]). Fekete et al. [22] first parallelized the McCaskill algorithm for a computer cluster. More recently, Palkowski and Bielecki [23] used the parallelizing source-to-source compilers PLUTO [24], [25] and TRACO [26] to automatically generate cache efficient and multi-core codes for the McCaskill algorithm.

In this paper, we begin by rewriting McCaskill's dynamic programming equations using a single matrix rather than two as used in the original equations. Then, using the row and box computation methods proposed by us in [27] and [28] to develop cache efficient algorithms for Nussinov's and Zuker's methods, respectively, for RNA folding, we develop new algorithms to compute the partition functions of McCaskill using a single array. The performance of our algorithms is compared experimentally with that of McCaskill's original algorithm and optimized versions obtained automatically by the optimizing compilers PLUTO and TRACO. Code for the original, PLUTO, and TRACO algorithms was obtained from [23]. Our experiments indicate that we are able to reduce run time by as much as $81.2 \%$ relative to the fastest previously known single core code and by as much as $84.7 \%$ relative to the fastest multi-core code using 20 threads on a 10 core Intel I9 CPU that supports hyperthreading.

The rest of the paper is organized in the following way. McCaskill's original dynamic programming equations and corresponding algorithm are presented in Section II. In Section III, we give our rewrite of McCaskill's equations and corresponding algorithms. Experimental results are presented in Section IV. Finally, Section V presents concluding remarks.

\section{MCCASKILL's EQUATIONS AND ALGORITHMS}

Let $A[1: n]=a_{1} a_{2} \cdots a_{n}$ be an RNA sequence. McCaskill [7] develops an $O\left(n^{4}\right)$ and an $O\left(n^{3}\right)$ algorithm to compute the partition functions of $A$. The $O\left(n^{3}\right)$ algorithm uses a simplified (constant) energy function and it is this algorithm that is the focus of [23] and this paper. Let $Q(i, j)$ be the partition function of the subsequence $A[i: j]$ and let $Q^{b p}(i, j)$ be the partition function of the subsequence $A[i: j]$ when $A[i]$ and $A[j]$ form a base pair $\left(Q^{b p}(i, j)\right.$ is 0 when $A[i]$ and $A[j]$ do not form a base pair). McCaskill's simplified dynamic programming equations are:

$$
Q_{i, i-1}=1,1 \leq i \leq n
$$




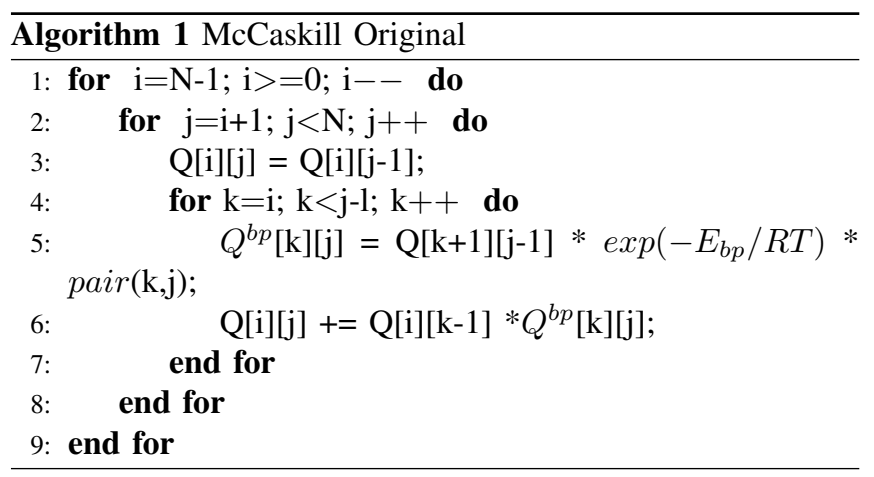

$$
\begin{gathered}
Q_{i, j}=Q_{i, j-1}+\sum_{i \leq k<j-l} Q_{i, k-1} * Q_{k, j}^{b p} \\
Q_{i, j}^{b p}=Q_{i+1, j-1} * \exp \left(-E_{b p} / R T\right) * \operatorname{pair}(i, j)
\end{gathered}
$$

where $\operatorname{pair}(i, j)$ is 1 if $A[i]$ and $A[j]$ are complementary base pairs such as $A U, G C$ and $G U$, and 0 otherwise; $E_{b p}$ is the fixed energy, contributed by a base pair; $R$ is a gas constant; $T$ is the temperature; and $l$ is the minimum loop length.

Using these equations, the partition functions $Q$ and $Q^{b p}$ may be computed using the algorithm Original (Algorithm 1) [23], which computes the $Q \mathrm{~s}$ and $Q^{b p} \mathrm{~s}$ by rows from bottom to top and within a row from left to right.

Palkowski and Bielecki [23] have experimented with optimized single core and multi core versions of Algorithm Original obtained using the source-to-source parallelizing compilers PLUTO [24], [25] and TRACO [26]. We use the terms Orig, Pluto, and Traco to refer to their single core single thread codes for the original algorithm and its optimization using PLUTO and TRACO, respectively.

\section{OUR ALgORITHMS}

\section{A. Base Algorithm For $Q$ Using One Triangular Array}

McCaskill's dynamic programming equations for $Q$ are easily rewritten as below by eliminating $Q^{b p}$ from Equation 2 using Equation 3. The rewritten equations are:

$$
\begin{gathered}
Q_{i, i-1}=1,1 \leq i \leq n \\
Q_{i, j}=Q_{i, j-1}+\sum_{\substack{i \leq k<j-l \\
* \exp \left(-E_{b p} / R T\right) * \operatorname{pair}(k, j)}} Q_{i, k-1} * Q_{k+1, j-1}
\end{gathered}
$$

$Q^{b p}(i, j)$, if needed, may be computed from $Q(i+1, j-1)$ using Equation 3 once all the $Q$ s have been computed.

Using the rewritten equations, $Q$ may be computed using a single array as shown in Algorithm 2 (OneArray). This algorithm computes $Q$ by diagonals and within a diagonal from top to bottom. It's run time is $O\left(n^{3}\right)$ and it uses $n(n+$ $1) / 2$ space when an upper triangular two-dimensional array [29] is used for $Q . Q^{b p}$ may be computed from $Q$ in $O\left(n^{2}\right)$ time using Equation 3.

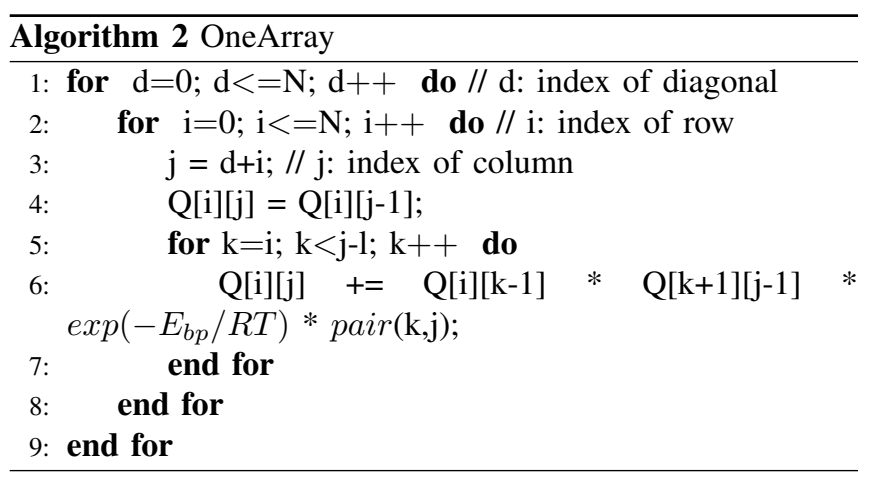

Notice that $Q \mathrm{~s}$ that are on the same diagonal may be computed simultaneously. So, algorithm OneArray is easily parallelized. Let OneArrayP be the parallel version of OneArray obtained by dividing each diagonal into $t$ tiles of approximately same size, where $t$ is the number of parallel threads.

\section{B. ByRow (ByRow Algorithm)}

In the loop of lines 5 and 6 of Algorithm OneArray (Algorithm 2), the memory accesses for $Q[k+1][j-1]$ are cache inefficient as these correspond to a column access while the memory accesses for $Q[i][k-1]$, which correspond to a row access, are cache efficient. As noted by us in [27], [28] cache efficiency is enhanced by computing the $Q$ s by rows bottom to top rather than by diagonals. Within a row, the computation is done left to right. Algorithm 3, ByRow, does exactly this. Though the computation order is the same in Algorithm ByRow as in Algorithm Original (Algorithm 1), there is a significant difference between the two algorithms besides the fact that Algorithm ByRow does not use $Q_{b p}$ explicitly. Once an element $Q[i][j]$ has been calculated in ByRow, all elements to its right which are on the same row are updated. This does not happen in Original. Relative to Algorithm 2, Algorithm 3 eliminates the inefficient column access for $Q$.

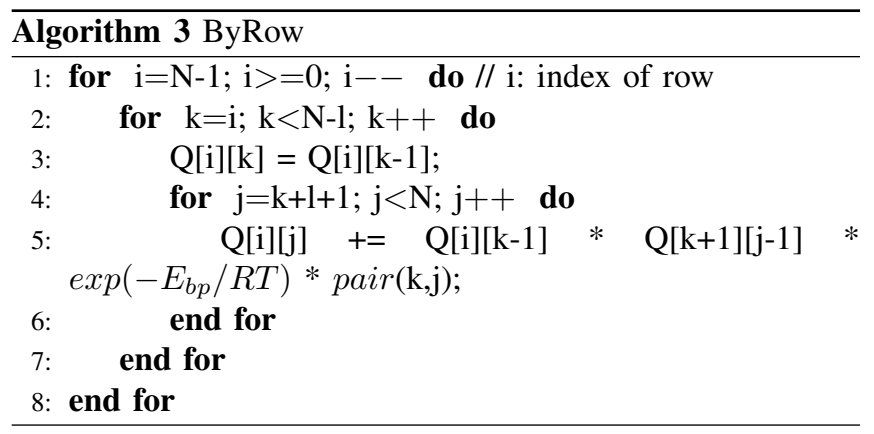

The innermost loop of Algorithm ByRow (i.e., the $j$ loop) is easily parallelized. The resulting parallel algorithm is called ByRowP.

\section{ByBox (ByBox Algorithm)}

The most cache efficient method developed by us for dynamic programming computes the elements in the upper 

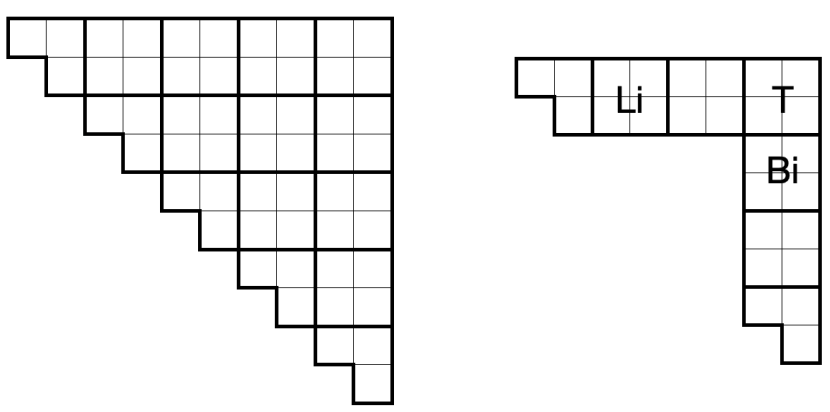

Fig. 1. Partitioning the upper triangle of $Q$ for computation by boxes

triangle of $Q$ by boxes rather than by rows [27], [28]. In this method, the upper triangle is divided into strips with $p$ rows each. Each strip is divided into $p \mathrm{x} p$ square boxes except the leftmost partition in each strip, which is a $p \times x$ triangular box (Figure 1). $p$ is chosen to be a multiple of the cache-line width $w . Q$ is computed by strips bottom to top. Within a strip, the computation is done by boxes left to right. The corresponding algorithm is ByBox (Algorithm 4).

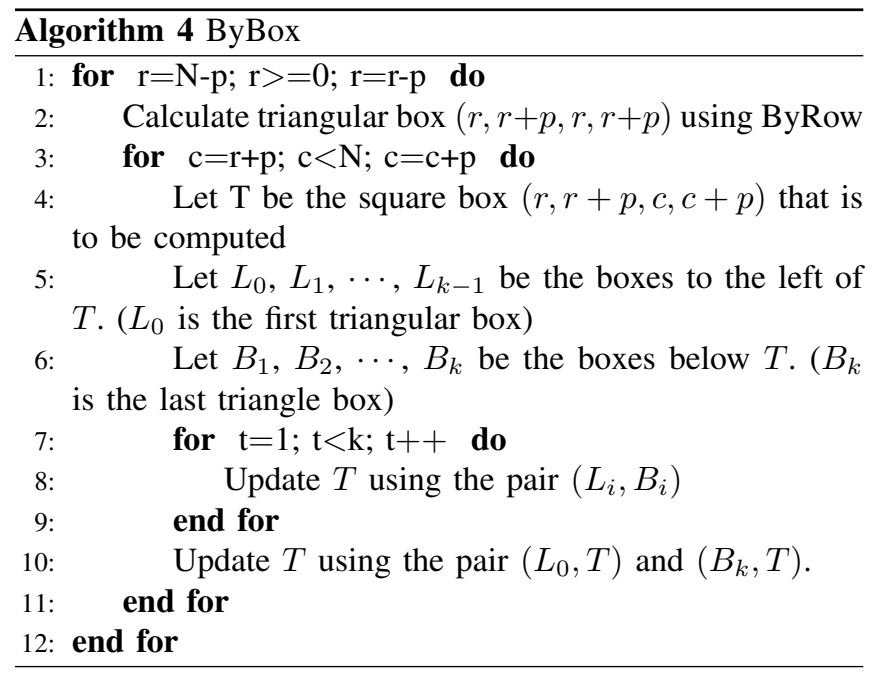

In our parallel version $B y B o x P$, we first compute the triangular blocks. Since these blocks are independent of one another, they may be computed in parallel using one processor per block. Next, the square blocks are computed one at a time bottom to top and within a strip left to right. When computing block $T$ (Figure 1), multiple block pairs $\left(L_{i}, B_{i}\right)$ can be handled simultaneously.

\section{EXPERIMENTAL RESULTS}

\section{A. Experimental Platforms and Test Data}

We implemented the single- and multi-core versions of the algorithms OneArray, ByRow, and ByBox of Section III in $\mathrm{C}$; openMP was used in the parallel codes. The performance of our codes was compared to that of the codes Orig, Pluto, and Traco obtained from [23]. The tile sizes for Pluto and Traco used by us were the defaults $(16 \times 16 \times 16)$ and $(1 \mathrm{x}$
$128 \times 16$ ) set by Palkowski et al. in these codes, respectively. For ByBox and ByBoxP, the box size $p$ was set to 32 . All codes were compiled using the gcc compiler with the O3 option and run on the platform: Intel I9-7900X Ten-Core processor $3.30 \mathrm{GHz}$ with $14 \mathrm{MB}$ LLC cache (Hyper threading supported).

For test data, we used RNA sequences obtained from the National Center for Biotechnology Information (NCBI) database [30].

\section{B. Performance on 19}

Table I gives the run times, in seconds, for the single core McCaskill algorithms on our I9 platform and shows the speedup obtained by ByBox relative to each of the single core single thread algorithms for each of our test sequences. Pluto is the fastest of the codes in [23] when run using a single thread and both ByRow and ByBox are consistently faster than Pluto. ByBox is the fastest of our codes. The run time reduction obtained by $B y B o x$ relative to Pluto ranges from $41.24 \%$ to $81.22 \%$ (column labeled $B$ vs $P$ ). $B y B o x$ achieves a speedup of up to approximately 5.3 relative to Pluto.

Table II gives the run times, in seconds, for the multi core McCaskill algorithms on our I9 platform. Times are given using both 10 threads and 20 threads. Traco was consistently faster with 20 threads than with 10 threads (recall that the I9 supports hyperthreading) and ByBoxP was faster with 20 threads for sequence sizes more than 4000 . ByBoxP was the fastest multicore algorithm on the $\mathrm{I} 9$ for all sequence sizes and Traco came in second. When 20 threads are used, ByBoxP takes between $57.36 \%$ and $84.76 \%$ less time than does Traco on our RNA sequences. Speedups of up to approximately 7.1 were obtained relative to Traco.

\section{CONCLUSION}

Using a rewrite of McCaskill's dynamic programming equation, we have obtained a one array implementation, OneArray, of McCaskill's algorithm to find the partition functions of an RNA sequence. Two cache efficient versions of OneArray along with parallel multi-core versions of all three of these algorithms have been developed. On our Intel I9 computational platform, our best single core algorithm, ByBox, takes up to $81.2 \%$ less time than the best single core algorithm in [23] and our best multi-core algorithm, ByBoxP, takes up to $84.7 \%$ less time than the best multi-core algorithm in [23].

\section{ACKNOWLEDGMENT}

This work was supported, in part, by the National Science Foundation under award 1447711.

\section{REFERENCES}

[1] M. S. Waterman and T. F. Smith, "RNA secondary structure: A complete mathematical analysis," Mathematical Biosciences, vol. 42, no. 3-4, pp. 257-266, 1978.

[2] R. Nussinov, G. Pieczenik, J. R. Griggs, and D. J. Kleitman, "Algorithms for loop matchings," SIAM Journal on Applied mathematics, vol. 35 no. 1 , pp. 68-82, 1978. 
TABLE I

RUN TIME FOR SINGLE CORE MCCASKILL ALGORITHMS, IN SECONDS, ON I9

\begin{tabular}{ll|lllllll}
\hline Seq & Size & Orig & Pluto & Traco & OneArray & ByRow & ByBox & $B$ vs $P$ \\
\hline AH001815.2 & 1,000 & 0.65 & 0.52 & 0.93 & 0.380 & 0.433 & 0.304 & $41.24 \%$ \\
XR_002696555.2 & 2,048 & 11.24 & 7.21 & 14.37 & 4.343 & 2.750 & 2.324 & $67.77 \%$ \\
NR_076111.2 & 3,048 & 44.37 & 29.51 & 50.48 & 24.152 & 10.476 & 7.773 & $73.66 \%$ \\
XR_002696557.1 & 4,026 & 128.87 & 82.39 & 162.11 & 65.872 & 20.427 & 18.281 & $77.81 \%$ \\
AJ966883.1 & 5,039 & 275.73 & 188.32 & 337.89 & 148.208 & 45.133 & 35.368 & $81.22 \%$ \\
\hline
\end{tabular}

TABLE II

RUN TIME FOR MULTI CORE MCCASKILL ALGORITHMS, IN SECONDS, ON I9

\begin{tabular}{|c|c|c|c|c|c|c|c|c|c|c|c|}
\hline \multirow[b]{2}{*}{ Seq } & \multirow[b]{2}{*}{ Size } & \multicolumn{5}{|c|}{10 threads } & \multicolumn{5}{|c|}{20 threads } \\
\hline & & Traco & OneArrayP & ByRowP & ByBoxP & $B$ vs $T$ & Traco & OneArrayP & ByRowP & ByBoxP & $B$ vs $T$ \\
\hline AH001815.2 & 1,000 & 0.15 & 0.05 & 1.03 & 0.08 & $47.73 \%$ & 0.15 & 0.04 & 1.48 & 0.06 & $57.36 \%$ \\
\hline XR_002696555.2 & 2,048 & 1.72 & 0.42 & 4.87 & 0.35 & $79.39 \%$ & 1.53 & 0.46 & 7.34 & 0.38 & $75.13 \%$ \\
\hline NR_076111.2 & 3,048 & 6.72 & 2.51 & 11.04 & 1.13 & $83.21 \%$ & 5.57 & 2.20 & 15.50 & 1.24 & $77.77 \%$ \\
\hline XR_002696557.1 & 4,026 & 18.37 & 7.16 & 21.02 & 2.60 & $85.83 \%$ & 15.54 & 6.35 & 30.60 & 2.51 & $83.85 \%$ \\
\hline AJ966883.1 & 5,039 & 37.47 & 16.51 & 31.09 & 4.87 & $87.00 \%$ & 31.27 & 14.02 & 44.98 & 4.77 & $84.76 \%$ \\
\hline
\end{tabular}

[3] M. Zuker and P. Stiegler, "Optimal computer folding of large RNA sequences using thermodynamics and auxiliary information," Nucleic acids research, vol. 9, no. 1, pp. 133-148, 1981.

[4] M. Zuker and D. Sankoff, "RNA secondary structures and their prediction," Bulletin of mathematical biology, vol. 46, no. 4, pp. 591-621, 1984.

[5] M. Zuker, "Computer prediction of RNA structure," in Methods in enzymology. Elsevier, 1989, vol. 180, pp. 262-288.

[6] - "On finding all suboptimal foldings of an RNA molecule," Science, vol. 244, no. 4900, pp. 48-52, 1989.

[7] J. S. McCaskill, "The equilibrium partition function and base pair binding probabilities for RNA secondary structure," Biopolymers: Original Research on Biomolecules, vol. 29, no. 6-7, pp. 1105-1119, 1990.

[8] S. Will, K. Reiche, I. L. Hofacker, P. F. Stadler, and R. Backofen, "Inferring noncoding RNA families and classes by means of genomescale structure-based clustering," PLoS computational biology, vol. 3, no. $4,2007$.

[9] I. L. Hofacker, S. H. Bernhart, and P. F. Stadler, "Alignment of RNA base pairing probability matrices," Bioinformatics, vol. 20, no. 14, pp 2222-2227, 2004.

[10] Z. J. Lu, J. W. Gloor, and D. H. Mathews, "Improved RNA secondary structure prediction by maximizing expected pair accuracy," Rna, vol. 15 , no. 10, pp. 1805-1813, 2009.

[11] M. Palkowski and W. Bielecki, "Parallel tiled cache and energy efficient codes for o (n4) RNA folding algorithms," Journal of Parallel and Distributed Computing, vol. 137, pp. 252-258, 2020.

[12] J. Li, S. Ranka, and S. Sahni, "Multicore and GPU algorithms for Nussinov RNA folding," BMC bioinformatics, vol. 15 , no. 8, p. S1, 2014.

[13] A. Mathuriya, D. A. Bader, C. E. Heitsch, and S. C. Harvey, "GTfold: a scalable multicore code for RNA secondary structure prediction," in Proceedings of the 2009 ACM symposium on Applied Computing, 2009 pp. 981-988.

[14] M. S. Swenson, J. Anderson, A. Ash, P. Gaurav, Z. Sükösd, D. A. Bader, S. C. Harvey, and C. E. Heitsch, "GTfold: Enabling parallel RNA secondary structure prediction on multi-core desktops," BMC research notes, vol. 5, no. 1, p. 341, 2012.

[15] G. Tan, N. Sun, and G. R. Gao, "A parallel dynamic programming algorithm on a multi-core architecture," in Proceedings of the nineteenth annual ACM symposium on Parallel algorithms and architectures, 2007, pp. 135-144.

[16] T. Estrada, A. Licon, and M. Taufer, "CompPknots: a framework for parallel prediction and comparison of RNA secondary structures with pseudoknots," in International Symposium on Parallel and Distributed Processing and Applications. Springer, 2006, pp. 677-686.
[17] F. Xia, Y. Dou, X. Zhou, X. Yang, J. Xu, and Y. Zhang, "Fine-grained parallel RNAalifold algorithm for RNA secondary structure prediction on FPGA," BMC bioinformatics, vol. 10, no. S1, p. S37, 2009

[18] A. Jacob, J. Buhler, and R. D. Chamberlain, "Accelerating Nussinov RNA secondary structure prediction with systolic arrays on FPGAs," in 2008 International Conference on Application-Specific Systems, Architectures and Processors. IEEE, 2008, pp. 191-196.

[19] Y. Dou, F. Xia, and J. Jiang, "Fine-grained parallel application specific computing for RNA secondary structure prediction using SCFGS on FPGA," in Proceedings of the 2009 international conference on Compilers, architecture, and synthesis for embedded systems, 2009, pp. 107 116.

[20] G. Rizk, D. Lavenier, and S. Rajopadhye, "GPU accelerated RNA folding algorithm," in GPU Computing Gems Emerald Edition. Elsevier, 2011, pp. 199-210.

[21] D.-J. Chang, C. Kimmer, and M. Ouyang, "Accelerating the Nussinov RNA folding algorithm with CUDA/GPU," in The 10th IEEE International Symposium on Signal Processing and Information Technology. IEEE, 2010, pp. 120-125.

[22] M. Fekete, I. L. Hofacker, and P. F. Stadler, "Prediction of RNA base pairing probabilities on massively parallel computers," Journal of Computational Biology, vol. 7, no. 1-2, pp. 171-182, 2000.

[23] M. Palkowski and W. Bielecki, "Parallel cache-efficient code for computing the mccaskill partition functions," in 2019 Federated Conference on Computer Science and Information Systems (FedCSIS). IEEE, 2019 pp. 207-210.

[24] U. Bondhugula, A. Hartono, J. Ramanujam, and P. Sadayappan, "A practical and fully automatic polyhedral program optimization system," in ACM SIGPLAN PLDI, vol. 10, no. 1375581.1375595, 2008.

[25] U. Bondhugula, M. Baskaran, S. Krishnamoorthy, J. Ramanujam, A. Rountev, and P. Sadayappan, "Automatic transformations for communication-minimized parallelization and locality optimization in the polyhedral model," in International Conference on Compiler Construction. Springer, 2008, pp. 132-146.

[26] M. Palkowski and W. Bielecki, "TRACO: source-to-source parallelizing compiler," Computing and Informatics, vol. 35, no. 6, pp. 1277-1306, 2017.

[27] C. Zhao and S. Sahni, "Cache and energy efficient algorithms for Nussinov's RNA folding," BMC bioinformatics, vol. 18, no. 15, p. 518, 2017.

[28] — , "Efficient RNA folding using Zuker's method," in 2017 IEEE 7th International Conference on Computational Advances in Bio and Medical Sciences (ICCABS). IEEE, 2017, pp. 1-6.

[29] S. Sahni, "Data structures, algorithms, and applications in c++, second edition." Silicon Press, 2005.

[30] "Ncbi database," http://www.ncbi.nlm.nih.gov/gquery. 